\title{
Nanomechanics of lignin-cellulase interactions in aqueous solutions
}

Peitao Zheng, ${ }^{a}, b,+$ Li Xiang, ${ }^{b, t}$ Jian Chang, ${ }^{c}$ Qiaojia Lin, ${ }^{a}$ Lei Xie, ${ }^{b}$ Tu Lan, ${ }^{b, d}$ Jing Liu, ${ }^{a}$ Zhenggang Gong, ${ }^{a}$ Tian Tang, ${ }^{d}$ Li Shuai, ${ }^{a}$ Xiaolin Luo, ${ }^{a, e, *}$ Nairong Chen, ${ }^{a, *}$ Hongbo Zeng ${ }^{b, *}$

${ }^{a}$ College of Material Engineering, Fujian Agriculture and Forestry University, 15

Shangxiadian Road, Fuzhou, Fujian, 350002, P. R. China.

${ }^{b}$ Department of Chemical and Materials Engineering, University of Alberta, Edmonton, Alberta, T6G 2V4, Canada.

${ }^{c}$ Department of Materials Science \& Engineering, Southern University of Science and Technology, Shenzhen 518055, China.

${ }^{d}$ Department of Mechanical Engineering, University of Alberta, Edmonton, Alberta T6G 1H9, Canada.

e Jiangsu Provincial Key Laboratory of Pulp and Paper Science and Technology, Nanjing Forestry University, Nanjing, Jiangsu, 210037, China.

*E-mail for corresponding authors: xluo@fafu.edu.cn (X. Luo), fafucnr@163.com (N.

Chen), hongbo.zeng@ualberta.ca (H.Zeng)

F Peitao Zheng and Li Xiang contributed equally to this work 


\section{Supplementary Methods}

\section{Lignin isolation}

According to method reported by previous research, ${ }^{1}$ the lignin used in this study (Figure 2, Figure 3, Figure 5, Figure S2 and Figure S3) was isolated from bamboo raw material by integrated method including ball milling, extensive enzymatic hydrolysis, mild acid hydrolysis, and liquid-liquid extraction. In general, bamboo powder (40 60 mesh) was freeze-dried and further grinded in a mill (ZM 200, Retsch, Haan, Germany) to pass a screen with a square opening size of $180 \mu \mathrm{m}$. Grinded bamboo power was further ball milled for $4 \mathrm{~h}$, while liquid nitrogen was initially added into ball-milling tank to avoid the effect of temperature on lignin structure. The carbohydrates in the resulted powder were hydrolyzed by the extensive enzymatic hydrolysis. The enzymatic hydrolysis was conducted with $2 \%(\mathrm{w} / \mathrm{v})$ bamboo power loading at $50{ }^{\circ} \mathrm{C}$ and $200 \mathrm{rpm}$ for $48 \mathrm{~h}$ and repeated for 3 times. The cellulase, cellobiase and xylanase (Pentopan Mono $B G \AA$, 2500U/g, Sigma-Aldrich, Shanghai, China) loadings for each time of enzymatic hydrolysis were, $30 \mathrm{FPU} / \mathrm{g}$ glucan, $45 \mathrm{CBU} / \mathrm{g}$ glucan and $10 \mathrm{mg}$ protein/g solid substrate (on o.d. basis), respectively. After 3 times of enzymatic hydrolysis, the slurry was filtered on a Büchner funnel. Resulted solid material was further washed by deionized water ( $2 \%$, $\mathrm{w} / \mathrm{v})$ and sodium phosphate buffer $(50 \mathrm{mM}, \mathrm{pH} 7.0,2 \%(\mathrm{w} / \mathrm{w}))$ at room temperature for $60 \mathrm{~min}$, respectively. Residual enzymes adsorbed on solid material were further hydrolyzed by protease $\left(2.4 \mathrm{U} / \mathrm{mL}\right.$, Sigma-Aldrich, Shanghai, China) at $50{ }^{\circ} \mathrm{C}$ and 200 $\mathrm{rpm}$ for $24 \mathrm{~h}$. The loadings of protease and crude lignin were fixed as $0.2 \mathrm{~mL} / \mathrm{g}$ lignin and 
$2 \%(\mathrm{w} / \mathrm{v})$, respectively. At the end of hydrolysis, the solid-liquid mixture was filtered on

a Büchner funnel. Resulted solid was further cleaned by $\mathrm{NaCl}$ solution $(200 \mathrm{~mL}, 1 \mathrm{M})$ and $200 \mathrm{~mL}$ deionized water at room temperature for $60 \mathrm{~min}$, respectively. By centrifugal separation and freezing-dry, the resulted crude lignin was refluxed in acidic dioxanewater $(85: 15, \mathrm{v} / \mathrm{v} ; 0.05 \mathrm{~mol} / \mathrm{L} \mathrm{HCl})$ solution (azeotrope boiling point of $86{ }^{\circ} \mathrm{C}$ ) under nitrogen for $2 \mathrm{~h}$. After that, the mixture was cooled to room temperature and centrifuged at $8000 \mathrm{rpm}$ for $15 \mathrm{~min}$. The resulting supernatant was neutralized with $\mathrm{NaHCO}_{3}(0.05$ $\mathrm{mol} / \mathrm{L}$ ) and concentrated at $30{ }^{\circ} \mathrm{C}$ through vacuum rotary evaporation. The concentrated solution was slowly dropped into $\mathrm{HCl}$ solution with a $\mathrm{pH}$ of 2 and gray solid was precipitated out. The mixture was centrifuged, washed with distilled water and freezingdried to obtain crude lignin sample.

In order to further remove the lignin-carbohydrate complex, the crude lignin separated from $10 \mathrm{~g}$ of bamboo powder was dissolved in $210 \mathrm{~mL}$ of pyridine-acetic acidwater $(9: 1: 4, \mathrm{v}: \mathrm{v}: \mathrm{v})$. The mixture was then extracted with $270 \mathrm{~mL} \mathrm{CHCl}_{3}$ at room temperature for $12 \mathrm{~h}$ and three times. The extracted organic layers were collected together and slowly dropped into $2000 \mathrm{~mL}$ of diethyl ether to make lignin precipitated. The precipitated lignin was washed with $200 \mathrm{~mL}$ diethyl ether to remove pyridine and then freezing-dried to obtain a relative pure sample. Finally, purified lignin was termed as milled bamboo lignin (MBL).

\section{Preparation of cellulose, cellulase and lignin films on mica}


An ultrathin cellulose film was prepared according to the previous report. ${ }^{2}$ Microcrystalline cellulose was initially silylated by hexamethyldisilazane and dissolved in toluene to prepare trimethylsilyl cellulose (TMSC) solution $(0.1 \mathrm{mg} / \mathrm{mL})$. Then TMSC solution was spinly coated (4000 rmp, $60 \mathrm{~s}$ ) on the surface of mica and hydrolyzed to form regenerated cellulose film by exposing to the vapor of $37 \%$ hydrochloric acid for 10 min. The surface of the cellulose film was analyzed using an X-ray photoelectron spectroscopy (XPS) (Thermo Fisher Scientific, USA). According to the previously reported method, ${ }^{1}$ isolated bamboo lignin was dissolved in pyridine/acetic acid/water $(9: 1: 4, \mathrm{v} / \mathrm{v} / \mathrm{v})$ with a concentration of $0.1 \mathrm{mg} / \mathrm{mL}$. Lignin solution was drop-coated on mica for $10 \mathrm{~min}$. After washing with the pyridine/acetic acid/water solution, lignin coating was washed five times with an acid-sodium acetate buffer (AA-SA, pH 5.0, 50 mmol/L). Commercial cellulase (Celluclast $1.5 \mathrm{~L}$ only) was diluted by AA-SA buffer solution to obtain a $0.1 \mathrm{mg}$ protein/mL enzyme solution and then drop-coated on the surface of mica. In general, $0.2 \mathrm{~mL}$ of prepared cellulase solution was dropped for 30 min. Finally, dropped mica was washed with AA-SA solution $(\mathrm{pH} 5.0,50 \mathrm{mmol} / \mathrm{L})$ for five times. The surface morphologies of dropped/coated mica were imaged by an atomic force microscope (Bruker, Santa Barbara, USA) operated in the tapping mode in the acetic acid-sodium acetate buffer solution ( $\mathrm{pH} 5.0,50 \mathrm{mmol} / \mathrm{L})$. The static water contact angle was determined using a contact angle goniometer (Ramé-hart, NJ, USA). Each measurement was conducted at least in triplicate. 


\section{QCM measurement}

The adsorption of cellulase on lignin film was performed using QCM (Q-Sense E1 instrument, Q-Sense AB, Sweden) according to the previous study. ${ }^{3,4}$ Prior to the test, a lignin solution was prepared by dissolving $0.1 \mathrm{~g} \mathrm{MBL}$ in $5 \mathrm{ml}, 0.75 \mathrm{~mol} / \mathrm{L}$ ammonium hydroxide, followed by centrifugation at $10000 \times \mathrm{g}$ for $10 \mathrm{~min}$. Centrifugation was performed twice to obtain completely dissolved lignin solution. QCM gold sensors were cleaned with a solution of water $/ 30 \%$ hydrogen peroxide $/ 25 \%$ ammonium hydroxide $(5: 1: 1, \mathrm{v} / \mathrm{v} / \mathrm{v})$ at $75^{\circ} \mathrm{C}$ for $30 \mathrm{~min}$ and rinsed thoroughly with abundant Milli-Q water, and then dried with nitrogen gas. The MBL solution was coated on the cleaned QCM gold sensor with a spin coater (WS-400Bz-6NPP-LITE, Mycro Technologies Corp., China) operated with three steps (1000 rpm for $30 \mathrm{~s}, 3000 \mathrm{rpm}$ for $60 \mathrm{~s}$ and $1000 \mathrm{rpm}$ for $30 \mathrm{~s}$ ). The obtained ultrathin lignin film was then exposed to the vapor of $37 \%$ hydrochloric acid for $30 \mathrm{~s}$ and dried with nitrogen gas. These films were immersed with Milli-Q water and dried with nitrogen gas again for QCM measurement. After obtaining the measurement baseline by injecting with AA-SA solution ( $\mathrm{pH} 5.0,1 \mathrm{mmol} / \mathrm{L})$ at a flow rate of $0.15 \mathrm{~mL}$ $\min ^{-1}$, the cellulase solution $(0.1 \mathrm{~g}$ protein $/ \mathrm{ml})$ with different cation concentration was introduced to measure the adsorption of cellulase on the lignin film.

\section{Water contact angle}


The water contact angles of different sample surfaces were determined by using a standard goniometer (Model 250, Ramé-hart, USA). The images of the sessile drops were fitted to determine the contact angle.

\section{Chemical pretreatments}

Lignocellulosic powder (bamboo or eucalyptus particles, 40 60 mesh) was pretreated by phenylsulfonic acid (PSA) or n-pentanol (PTL) pretreatments to deconstruct its recalcitrant structure. For PSA pretreatment, the PSA was pre-dissolved in deionized water at $50{ }^{\circ} \mathrm{C}$, in which the PSA concentration was $80 \%(\mathrm{w} / \mathrm{w})$. Some lignocellulosic powder and PSA solution were successively added into the round-bottom flask to yield a 15:1 (w/w) mass ratio of the PSA solution to the bamboo powder. The round bottom flask was placed in the oil bath to conduct the pretreatment at $90{ }^{\circ} \mathrm{C}$ for $30 \mathrm{~min}$. During the pretreatment, the bamboo power was magnetically stirred. At the end of pretreatment, the round-bottom flask was immediately cooled by tap water. The resulted slurry was filtered on a Büchner funnel and then washed 3 times with triple volumes of deionized water to remove the solvent from the pretreated solid substrate. Washed solid substrates were stored in a refrigerator at $4{ }^{\circ} \mathrm{C}$ for subsequent enzymatic hydrolysis.

PTL pretreatment was conducted in a microwave reactor at $125^{\circ} \mathrm{C}$ for $25 \mathrm{~min}$. The volumetric content of PTL in water and the ratio of pretreatment solution to lignocellulosic powder were fixed as $80 \%(\mathrm{v} / \mathrm{v})$ and 6: $1(\mathrm{ml}: \mathrm{g})$, respectively. Sulfuric acid loading was $4 \mathrm{wt} \%$ based on the o.d. weight of lignocellulosic powder. Other 
procedures for PTL pretreatment were same to that of PSA pretreatment.

\section{Compositional analysis}

Pretreated solid substrates were vacuum-dried at $40{ }^{\circ} \mathrm{C}$ overnight to make the moisture content lower than 10\%. The contents of Klason lignin and glucan in dried substrates were further measured by a reported method developed by the US National Renewable Energy Laboratory ${ }^{5}$. The glucan contents were measured as $82.4 \%, 85.3 \%$ and $82.5 \%$ for PSA pretreated bamboo and eucalyptus substrates, and PTL pretreated bamboo substrate, while the corresponding Klason lignin contents were $5.9 \%, 6.4 \%$ and $8.1 \%$, respectively. The Klason lignin content of MBL was also determined as $92.6 \%$ accordingly.

\section{Enzymatic hydrolysis}

Water-soluble SP was pre-extracted from defatted soybean powder (DSF) with AASA buffer solution. $10 \mathrm{~g}$ (o.d.) of DSF was mixed with 50-mL AA-SA buffer solution in a flask. The flask was shaken at $50{ }^{\circ} \mathrm{C}$ for $2 \mathrm{~h}$ on a shaker and then ultrasonically treated at $300 \mathrm{~W}$ for $30 \mathrm{~min}$. The supernatant containing water-soluble SP was separated through centrifuging the resultant slurry at a speed of $5000 \mathrm{rpm}$ for $5 \mathrm{~min}$. The SP concentration of supernatant was measured according to a reported bicinchoninic acid (BCA) method. ${ }^{6}$

Without treatment, the microcrystalline cellulose (Avicel) was directly subjected to enzymatic hydrolysis. All enzymatic hydrolysis of Avicel were conducted in a shaker with a $2 \%(\mathrm{w} / \mathrm{v})$ solid substrate loading in an AA-SA buffer $(\mathrm{pH} 5.0,50 \mathrm{mmol} / \mathrm{L})$ at 150 
rpm and $50{ }^{\circ} \mathrm{C}$ for $72 \mathrm{~h}$. For the enzymatic hydrolysis without SP, the Avicel, enzymes (cellulase and cellobiase), MBL and tetracycline solutions were simultaneously mixed in AA-SA buffer solution. The ratio of cellulase (Celluclast $1.5 \mathrm{~L}^{\circledR}$ ) and cellobiase (Novozyme 188) loadings for all enzymatic hydrolysis experiments were 1:2 (i.e., $5 \mathrm{FPU} / \mathrm{g}$ glucan (o.d.) for cellulase and $10 \mathrm{CBU} / \mathrm{g}$ glucan (o.d.) for cellobiase, respectively). For the enzymatic hydrolysis with SP as lignin-shield agent, the Avicel, MBL, tetracycline solutions, SP and AA-SA buffer solution were pre-mixed in a shaker at $150 \mathrm{rpm}$ and $50{ }^{\circ} \mathrm{C}$ for $2 \mathrm{~h}$. After that, the enzymes were added into the buffer solution and the enzymatic hydrolysis time was started to record. The mass ratio of MBL to Avicel ranges from 0 to $2(\mathrm{~m} / \mathrm{m})$. The cellulase (Celluclast $1.5 \mathrm{~L} ®)$ and cellobiase (Novozyme 188) loadings were $5 \mathrm{FPU} / \mathrm{g}$ glucan (o.d.) and $10 \mathrm{CBU} / \mathrm{g}$ glucan (o.d.), respectively. The tetracycline concentration in enzymatic system was $50 \mathrm{mmol} / \mathrm{L}$. At the end of the enzymatic hydrolysis, the hydrolysate was sampled. A glucose analyzer (2900D, YSI Inc., Yellow Springs, OH, USA) was used to determine the concentration of glucose in the enzymatic hydrolysates. The following equation was used to calculate the enzymatic conversion $(\mathrm{EC})$ of glucan in cellulose or pretreated substrate.

$$
\mathrm{EC}=\frac{0.9 W_{\text {Glucose }}}{W_{\text {cellulose }}}
$$

where $W_{\text {Glucose }}$ is the amount of glucose released in the enzymatic hydrolysate (g); $W_{\text {Cellulose }}$ is the weight of cellulose (g) added into enzymatic hydrolysis system; 0.9 is the conversion coefficient of glucose to glucan during enzymatic hydrolysis. 
Without the addition of MBL, the conditions of enzymatic hydrolysis for the pretreated bamboo or eucalyptus substrate were same to that of Avicel. The SP was also pre-mixed with the pretreated substrate for $2 \mathrm{~h}$. 


\section{Supplementary Results}

a

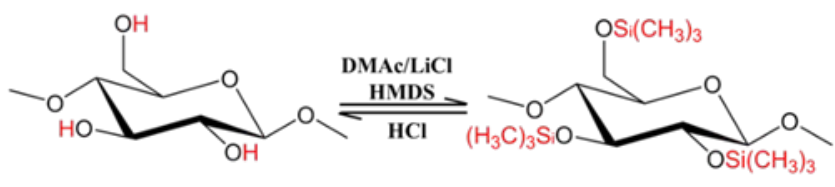

b
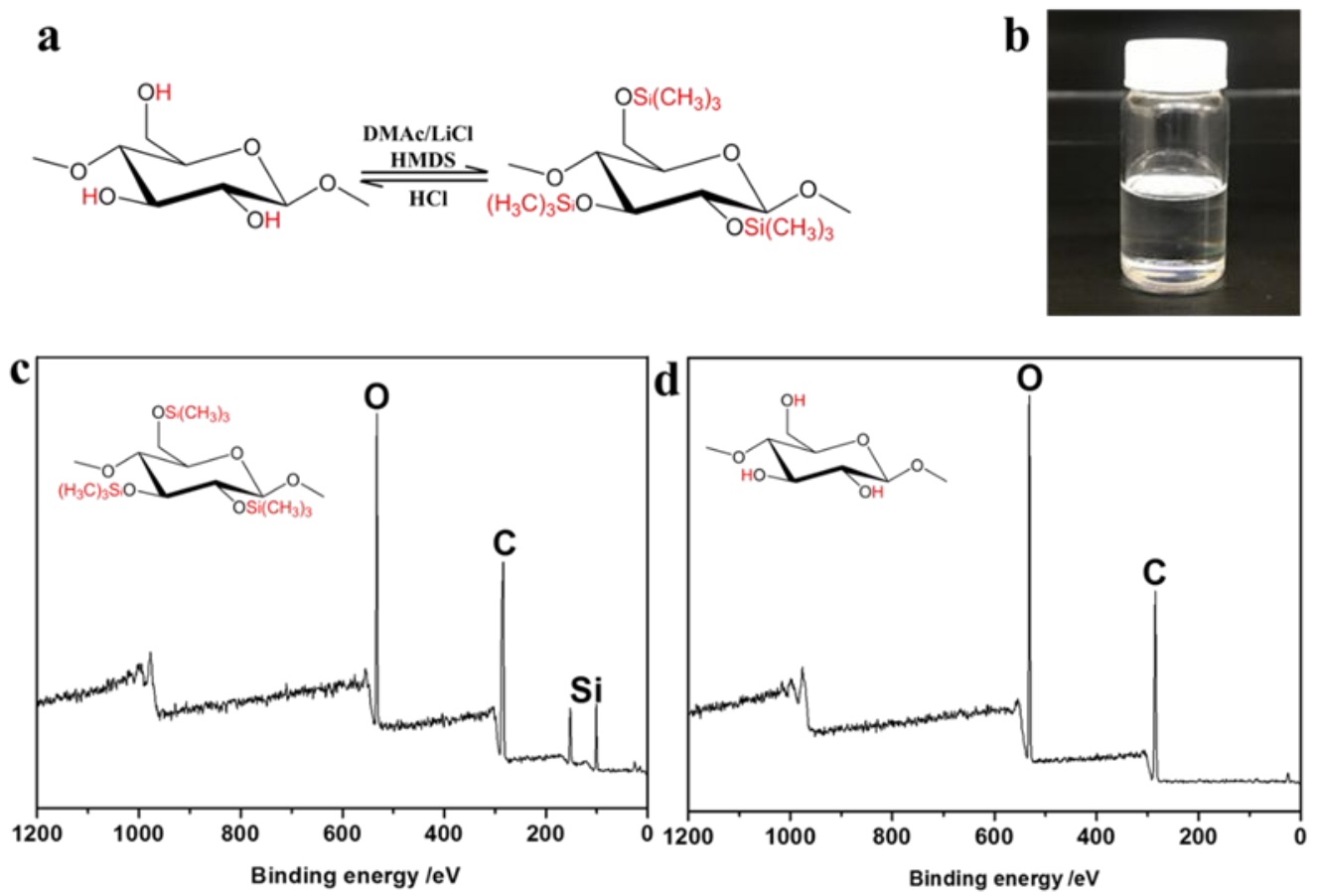

Figure S1. (a) Cellulose derivatization reaction formula, (b) trimethylsilyl cellulose solution, (c) XPS spectra of trimethylsilyl cellulose and (d) regenerated cellulose

A relative smooth cellulose film was prepared according to the foregoing study by a salinization-derived method. ${ }^{7}$ In general, the cellulose was firstly silylated by hexamethyldisilazane (HDMS) to trimethylsilyl cellulose (TMSC) and then hydrolyzed to form regenerated cellulose (Figure S1a). The strong silicon peak appears on the XPS spectra of TMSC (Figure S1c), implying that the silyl groups were successfully grafted on cellulose. Such derivatization makes the TMSC completely dissolved in the toluene solution (Figure S1b) and also provides the firmly bonding with mica. ${ }^{2}$ The spin-coated TMSC film was then hydrolyzed by hydrochloric acid, leading to the silicon peak completely disappeared (Figure S1d), 
indicating that the silyl groups were successfully hydrolyzed to the hydroxyl groups. This result is consistent with the previous report by Kontturi et al. ${ }^{2}$ For cellulase and lignin, the coatings (Experimental section, Preparation of cellulose, cellulase and lignin film on mica) were carried out in acetic acid-sodium acetate buffer $(\mathrm{pH} 5.0,50 \mathrm{mmol} / \mathrm{L})$ and pyridine-acetic acid-water (9:1:4,v: v: v), respectively.
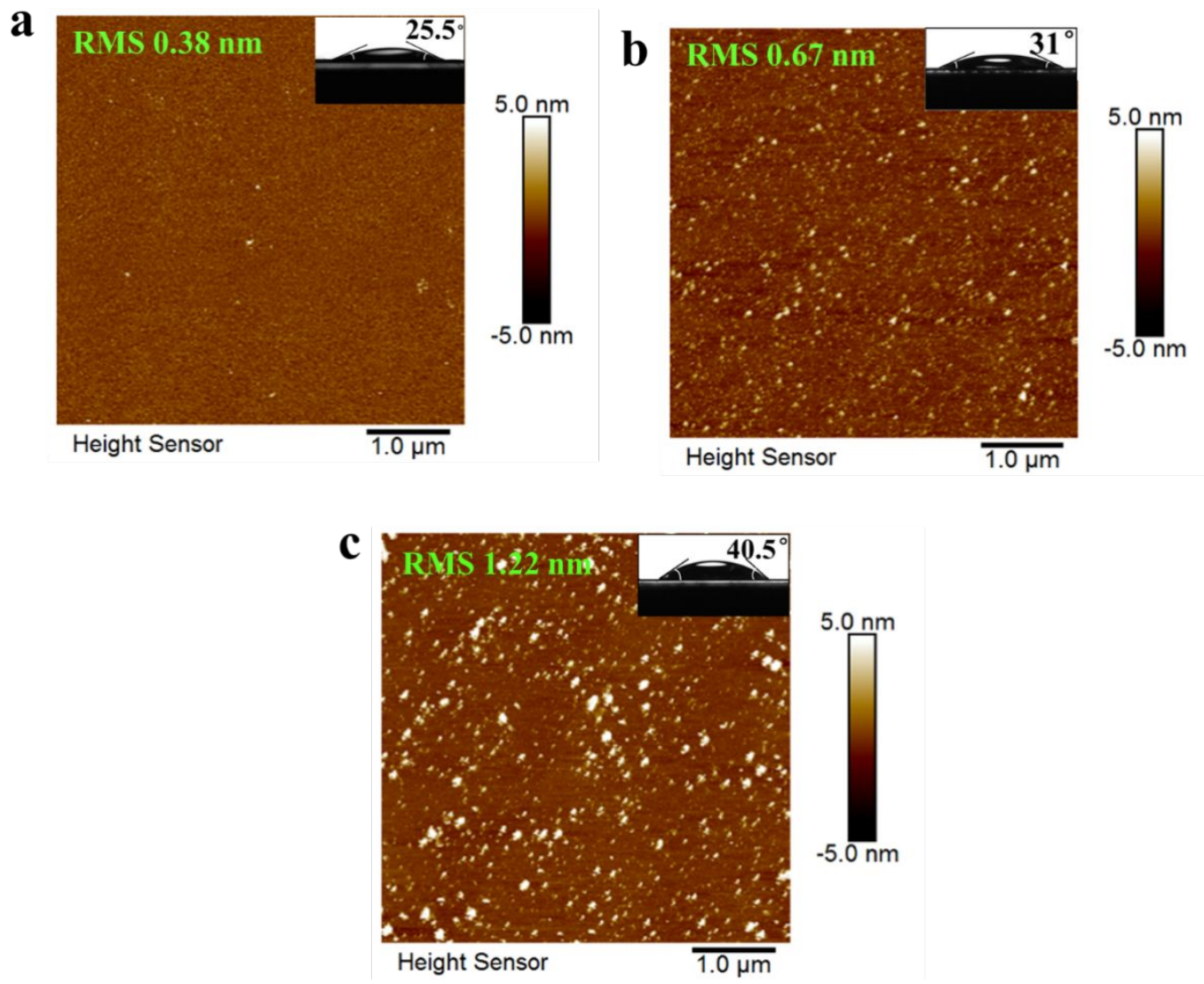

Figure S2. The contact angle and AFM images of cellulose (a), cellulase (b) and lignin (c)

coating 


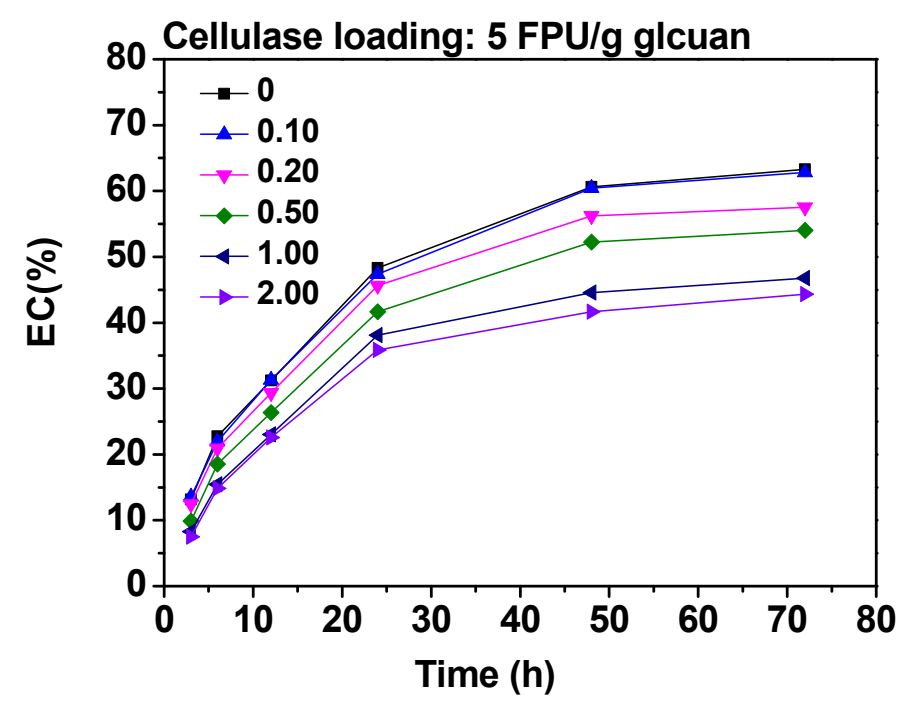

Figure S3. The time-dependent enzymatic conversions (ECs) of microcrystalline cellulose (Avicel) with the different lignin additions. The mass ratio of lignin to Avicel ranges from 0 to 2.

As shown in Figure S3, the isolated lignins decreased the ECs of microcrystalline cellulose (Avicel) from $63.3 \%$ to $44.3 \%$ with the ratio of lignin to cellulose $(\mathrm{w} / \mathrm{w})$ increasing from 0 to 2 . As a result, more cellulases are required to achieve a decent enzymatic hydrolysis yield, which inevitably increases the cost of enzymatic hydrolysis and makes the whole conversion process economically inviable. 
(a)

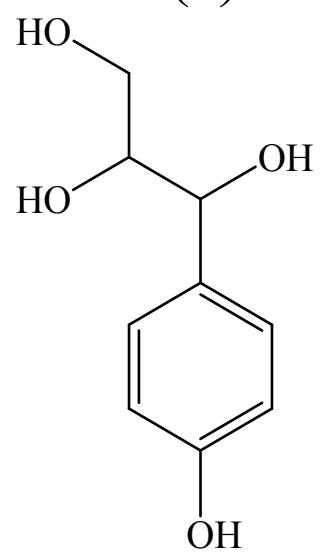

(b)<smiles>CC[N+](CC)(CC)CC</smiles>

Figure S4. (a) Molecular structures of a lignin model and (b) TEA employed in this work. 


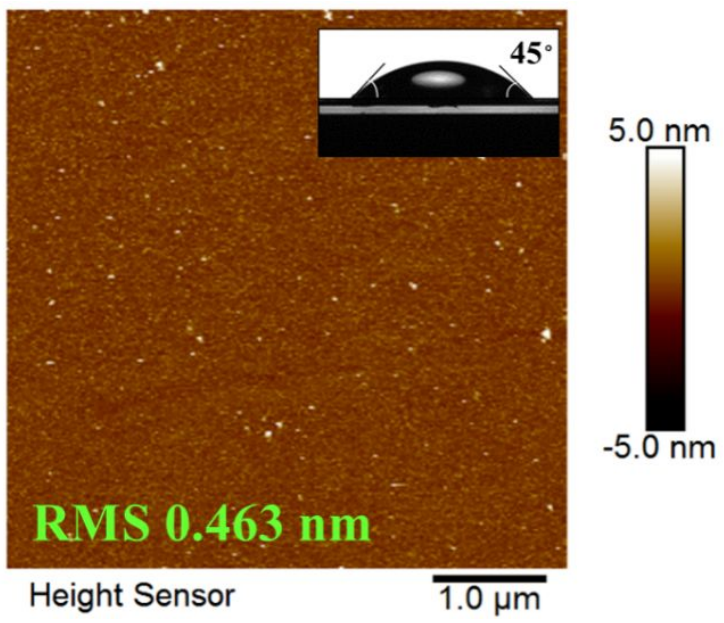

Figure S5. The contact angle and AFM images of SP 

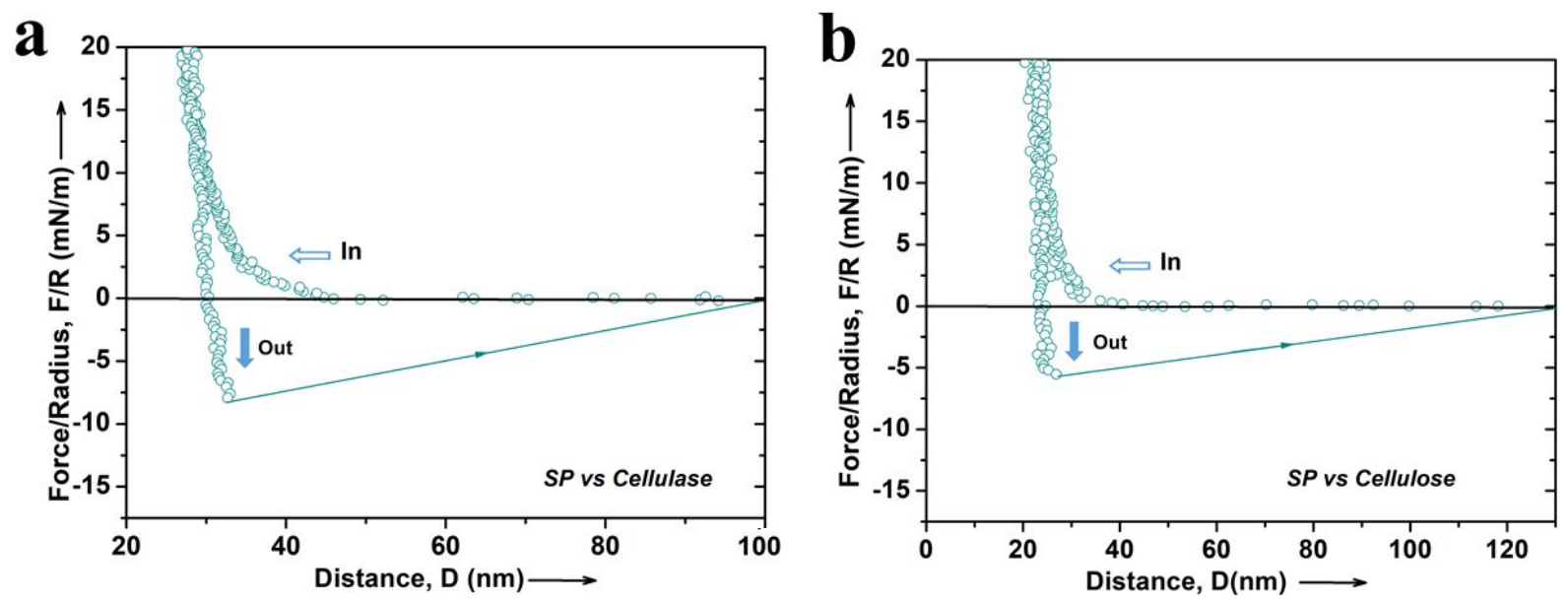

Figure S6. Typical force-distance ( $F / R$ vs. $D$ ) profiles of SP-Cellulose and SP-cellulase in initial buffer solution $\left(1 \mathrm{mM} \mathrm{Na}^{+}, \mathrm{pH} 5.0\right)$. 


\section{References}

1. Argyropoulos, D. S.; Sun, Y.; Palus, E., Isolation of Residual Kraft Lignin in High Yield and Purity. J. Pulp Pap. Sci. 2002, 28 (2), 50-54.

2. Kontturi, E.; Thune, P. C.; Niemantsverdriet, J. W., Novel Method for Preparing Cellulose Model Surfaces by Spin Coating. Polymer 2003, 44 (13), 3621-3625.

3. Suchy, M.; Linder, M. B.; Tammelin, T.; Campbell, J. M.; Vuorinen, T.; Kontturi, E., Quantitative Assessment of the Enzymatic Degradation of Amorphous Cellulose by Using a Quartz Crystal Microbalance with Dissipation Monitoring. Langmuir 2011, 27 (14), 8819-8828.

4. Cai, C.; Zhan, X.; Zeng, M.; Lou, H.; Qiu, X., Using Recyclable pH-responsive Lignin Amphoteric Surfactant to Enhance the Enzymatic Hydrolysis of Lignocelluloses. Green Chem. 2017, 19 (22), 5479-5487.

5. Sluiter, A.; Hames, B.; Ruiz, R.; Scarlata, C.; Sluiter, J.; Templeton, D.; Crocker, D., Determination of Structural Carbohydrates and Lignin in Biomass, Laboratory Analytical Procedure (LAP), Technical Report: NREL/TP-510-42618, 2012. (available at https://www.nrel.gov/docs/gen/fy13/42618.pdf)

6. Smith, P. K.; Krohn, R. I.; Hermanson, G. T.; Mallia, A. K.; Gartner, F. H.; Provenzano, M. D.; Fujimoto, E. K.; Goeke, N. M.; Olson, B. J.; Klenk, D. C., Measurement of protein using bicinchoninic acid. Anal Biochem 1985, 150 (1), 76-85.

7. Osterberg, M., The Effect of a Cationic Polyelectrolyte on the Forces between Two Cellulose Surfaces and between One Cellulose and One Mineral Surface. J Colloid Interface Sci 2000, 229 (2), 620-627. 